\title{
Shielding Effectiveness Measurements Using Mode-Stirred Chambers: A Comparison of Two Approaches
}

\author{
MICHAEL O. HATFIELD
}

\begin{abstract}
This paper presents two methodologies for performing shielding effectiveness measurements using mode-stirred chambers. The first method, which utilizes the traditional single (discrete) frequency approach, has been used at the Naval Surface Warfare Center (NSWC) for several years and is referred to as the discrete frequency method. By contrast, the second method utilizes broad-band noise sources and can measure shielding effectiveness over large bandwidths with high resolution in short periods of time. The second method is referred to as the noise method.

Index Code-F1b/f/g.
\end{abstract}

\section{INTRODUCTION}

C OMMONLY used shielding effectiveness measurement techniques such as described in MIL-STD-285 are time consuming and lacking in repeatibility [1]. For the past six years the Naval Surface Warfare Center (NSWC) in Dahlgren, Virginia, has been pursuing the development of techniques by which mode-stirred chambers are used to perform various types of electromagnetic evaluations. Shielding effectiveness measurements using the mode-stirred chamber have proven to be fast, easy to perform, and repeatable.

This paper describes and compares two methods of performing radiated shielding effectiveness measurements. The first method is referred to as the "discrete frequency method" and, as the name implies, involves testing one frequency at a time. The second method is referred to as the "noise method" and utilizes broad-band noise sources to create a continuous spectrum of test frequencies. In both cases, a mode-stirred chamber is used to contain and uniformly distribute the fields incident on the equipment under test (EUT). A brief description of mode-stirred chambers is given; however, this paper focuses on the comparison of the discrete frequency and noise methods and does not include a detailed discussion on the theory and operation of mode-stirred chambers. Readers not acquainted with mode-stirred chambers are urged to read [2].

\section{Mode-Stirred Chamber Description}

\section{A. General Description}

A mode-stirred chamber is simply a modified shielded room. For some applications, even small metal enclosures, e.g., a $1 \times 1 \times 2 \mathrm{~m}$ box, will work quite well. However, the size of the chamber does restrict the lowest frequency at which testing can be conducted. To qualify for use as a mode-

Manuscript received October 1.1987

The author is with the Naval Surface Warfare Center, Dahlgren, VA 22448. Tel. (703) 663-8594.

IEEE Log Number 8821928 stirred chamber an enclosure must be a conductive RF-tight enclosure with the smallest dimension being large compared to the operating wavelength. This frequency-size relationship imposes a lower frequency limitation, generally restricting operation to frequencies higher than $200 \mathrm{MHz}$ for most shielded rooms. A unique feature of a mode-stirred chamber is the "paddle-wheel" tuner, a relatively large field-perturbing device that is rotated within the chamber. This irregularly shaped metal structure causes very large changes in the standing-wave patterns. In this way, the many simultaneously existing modes are "stirred" just as in the case of a microwave oven.

\section{B. NSWC Chamber Description}

The NSWC mode-stirred chamber, shown in Figs. 1-3, is a large continuously welded steel enclosure measuring $10.82 \mathrm{~m}$ (L) $\times 5.18 \mathrm{~m}(\mathrm{~W}) \times 3.96 \mathrm{~m}(\mathrm{H})$. If desired, the chamber can be divided into two separate enclosures by a removable double-walled bulkhead so that the two smaller chambers are: 1) $5.79 \mathrm{~m}(\mathrm{~L}) \times 5.18 \mathrm{~m}(\mathrm{~W}) \times 3.96 \mathrm{~m}(\mathrm{H})$, and 2) $4.72 \mathrm{~m}(\mathrm{~L})$ $\times 5.18 \mathrm{~m}(\mathrm{~W}) \times 3.96 \mathrm{~m}(\mathrm{H})$.

Fields in the NSWC chamber are established by means of an RF source driving: 1) solid-state amplifiers at frequencies from $200 \mathrm{MHz}$ to $1 \mathrm{GHz}$, and 2) TWT amplifiers at frequencies from 1 to $18 \mathrm{GHz}$. Each amplifier is connected to a transmitting antenna mounted inside the chamber. Logperiodic antennas are used from $200 \mathrm{MHz}$ to $1 \mathrm{GHz}$ and rectangular horn antennas, matched in bandwidth to each TWT, are used from 1 to $18 \mathrm{GHz}$.

Each of the chamber sections is equipped with a fieldperturbing device previously referred to as a paddle wheel. The paddle wheel shown in Fig. 4 is a large $(1 \mathrm{~m} \times 2 \mathrm{~m} \times 2$ $\mathrm{m})$ reflecting surface suspended from the chamber ceiling. Fields inside the chamber are "stirred" by the paddle wheels by means of stepper motors which allow the paddle wheels to be stepped in increments as small as $0.1125^{\circ}$ (3200 steps per revolution). The dwell time between steps can be adjusted from milliseconds to hours, depending on the EUT and/or monitoring equipment response time. Analog motion can be approximated by selecting small paddle-wheel steps with short dwell times between steps.

\section{Shielding Efffectiveness Measuremfnts}

\section{A. Test Fixtures}

Shielding effectiveness measurements using a mode-stirred chamber can be made on cables [3], connectors (MIL-STD- 


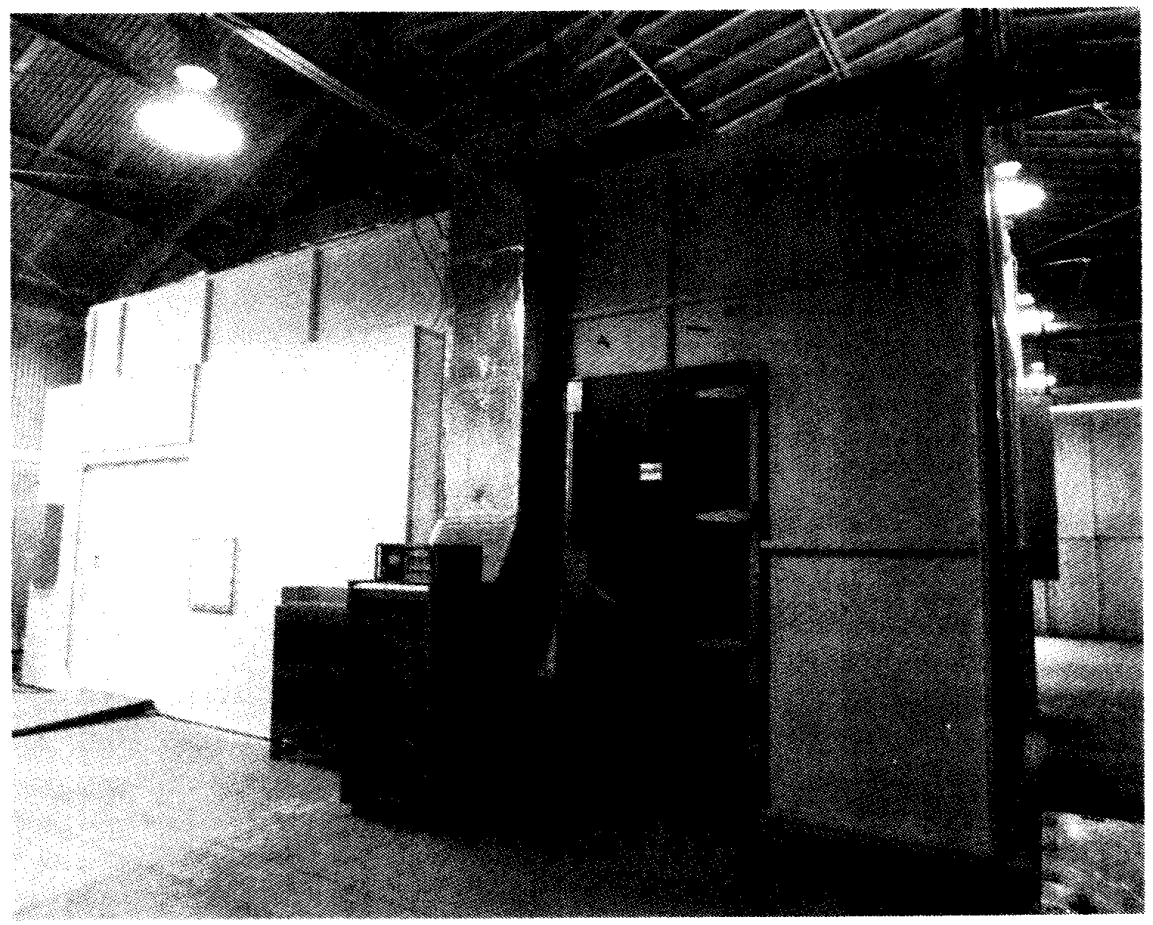

Fig. 1. Chamber exterior view.

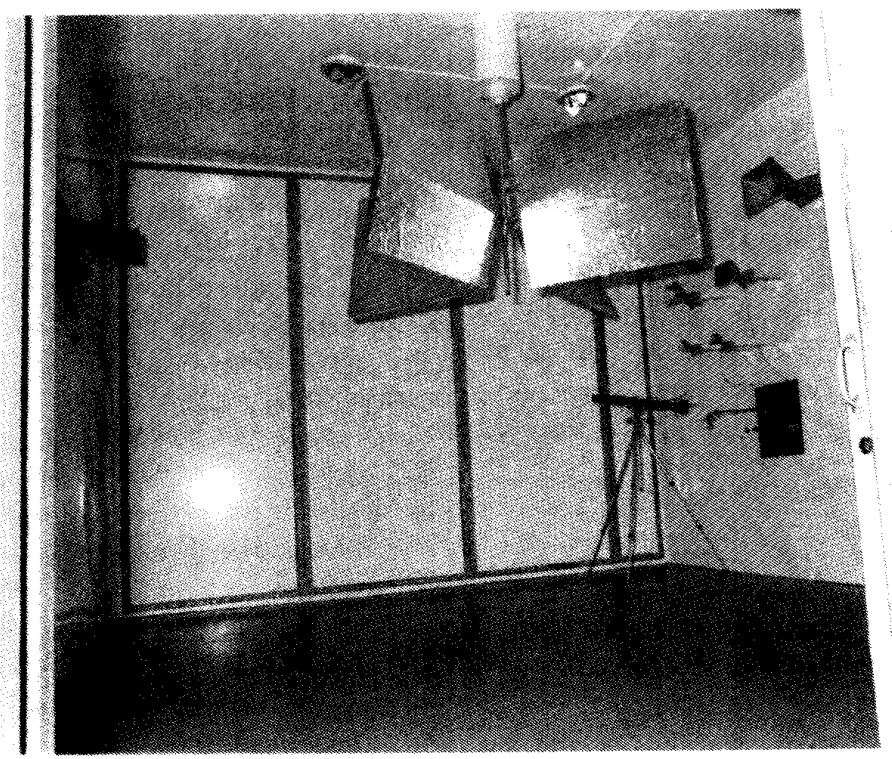

Fig. 2. Chamber interior view. 


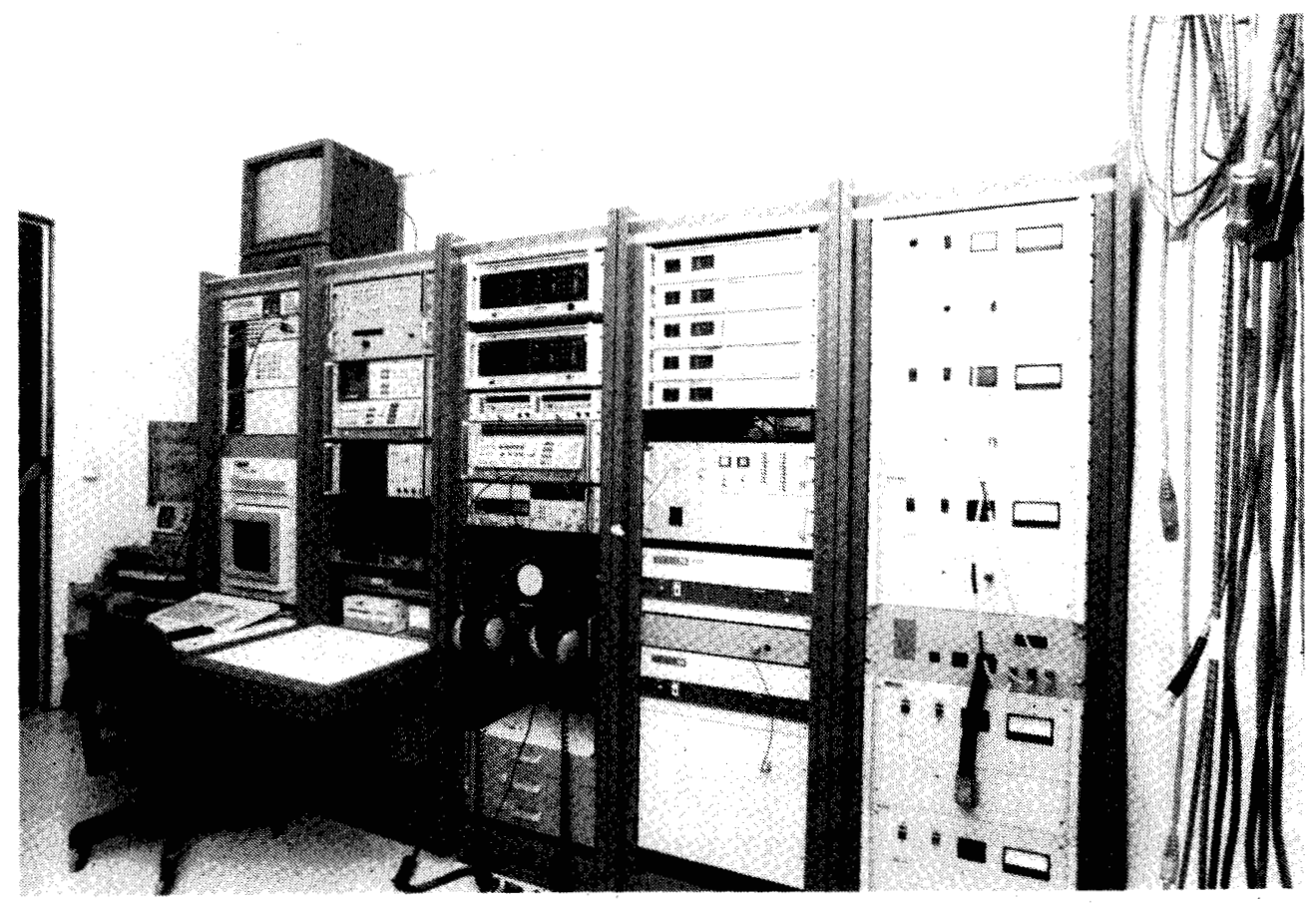

Fig. 3. Instrumentations for NSWC mode-stirred chamber.

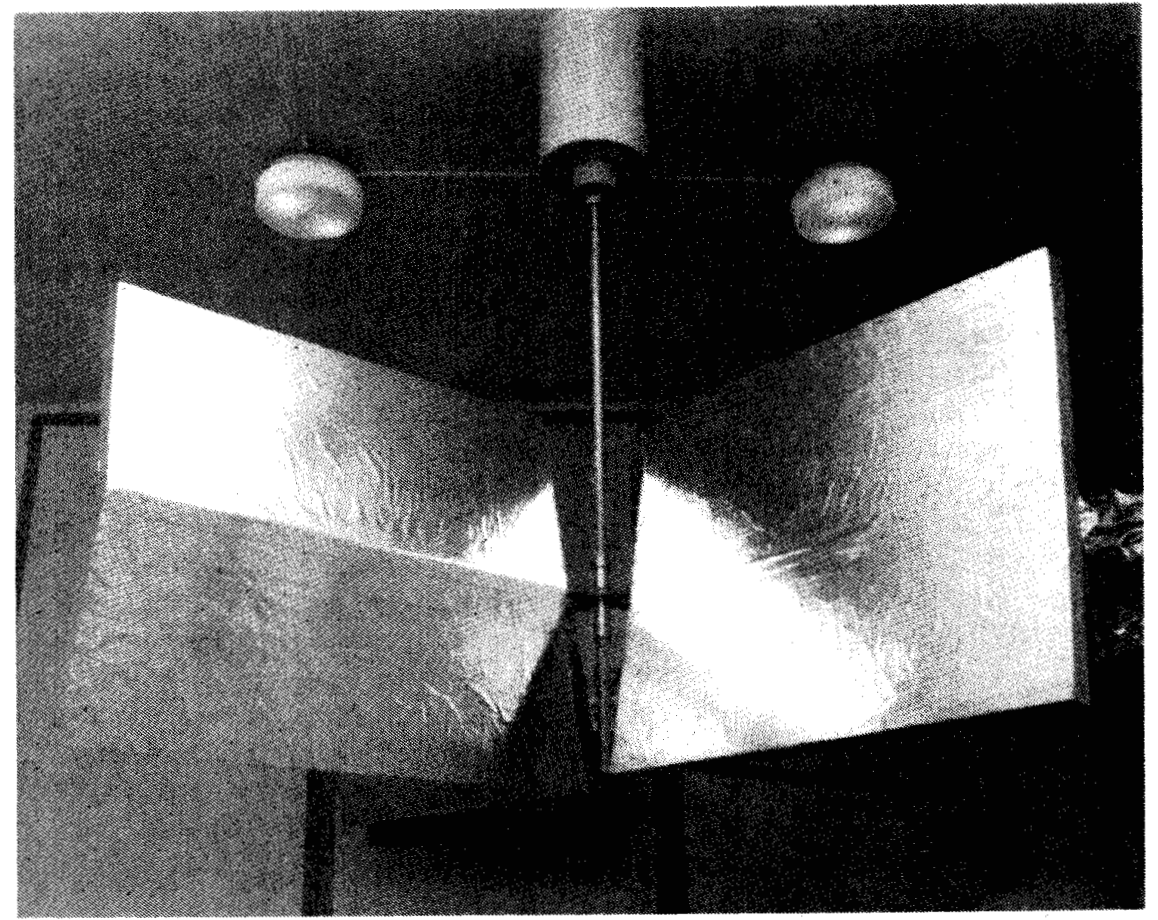

Fig. 4. Paddle-wheel close-up 


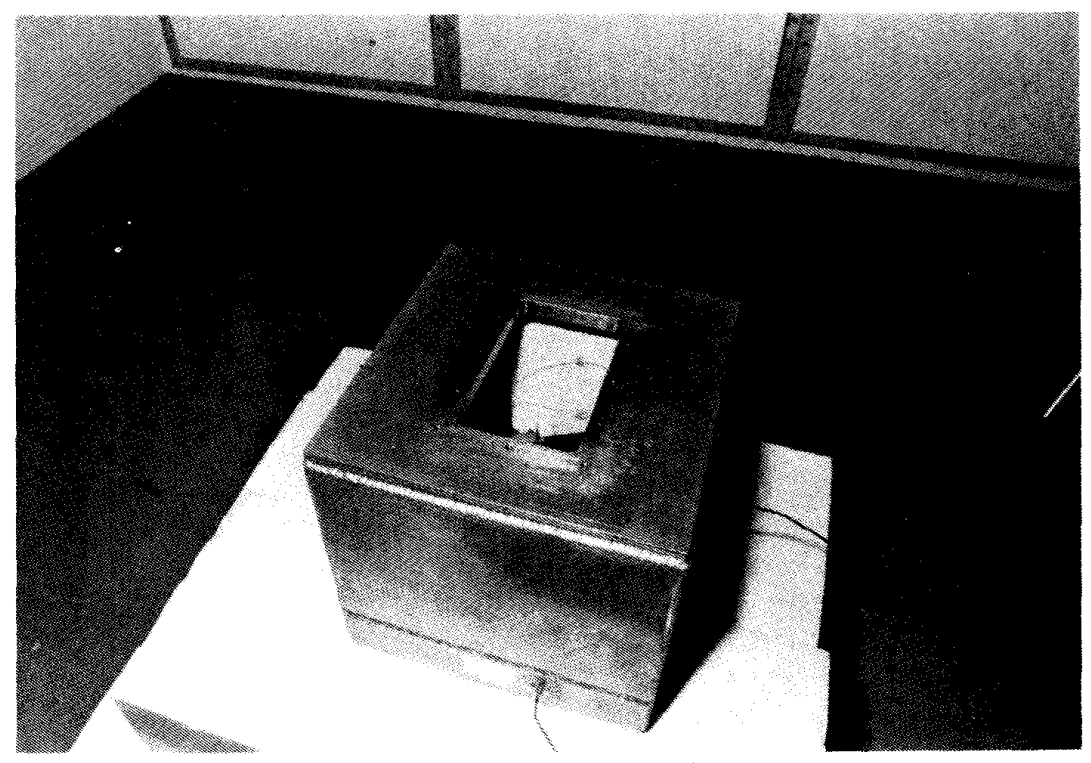

Fig. 5. Exterior view of a test fixture.

1344), materials, gaskets, or enclosures, to name a few. Items such as materials, gaskets, and enclosures utilize a "nested", chamber approach, i.e., a "mode-stirred chamber within a mode-stirred chamber." If the EUT is an enclosure, a paddle wheel and receiving antenna are installed inside the EUT, turning it into a small mode-stirred chamber. The internal paddle wheel ensures that any RF energy which "leaks" into the EUT will be detected. If the EUT is a material sample or gasket, a special test fixture must be built in which a paddle wheel and receiving antenna can be installed. The internal paddle is driven by a small $\mathrm{dc}$ motor and rotated at a constant speed, thereby stirring the fields inside the test fixture or enclosure. The speed at which the internal paddle wheel is rotated is adjusted to ensure that the monitoring equipment has time to respond to any leakage inside the EUT. The external paddle wheel is rotated $360^{\circ}$ in 200 or more steps per revolution [2]. The time interval between steps of the external paddle wheel must be greater than or equal to the time required for the internal paddle wheel to make one complete revolution.

The EUT for this test, a screen window with monel wire mesh and gasket, required a test fixture. The fixture, shown in Fig. 5, consisted of an aluminum box measuring $45.7 \mathrm{~cm} \times$ $45.7 \mathrm{~cm} \times 45.7 \mathrm{~cm}$. The test fixture was sectioned into two compartments to provide isolation between the receiving antenna, which was mounted in the larger upper compartment, and the dc motor, which was mounted in the lower compartment. The drive shaft of the dc motor protruded into the upper compartment to which the paddle wheel was mounted. A 25.4$\mathrm{cm} \times 15.2-\mathrm{cm}$ opening in the top of the test fixture served as an aperture over which the EUT could be mounted. The test fixture aperture, internal paddle wheel, and antenna are shown in Fig. 6.

The EUT, measuring $17.8 \mathrm{~cm} \times 10.2 \mathrm{~cm}$, required an adaptor plate in order to cover the aperture in the test fixture. The EUT was attached to the adaptor plate using fourteen 6-32 UNC screws as shown in Fig. 7, and remained attached to the adaptor plate for the duration of the test. This was done to prevent gasket wear and mounting repeatability associated with the EUT from affecting the test. Mounting differences and gasket wear associated with the mounting plate could also affect the test; however, any leakage associated with the adaptor plate would be detected during data collection.

\section{B. Data Collection}

The following three measurements must be made in order to ensure valid shielding effectiveness measurements.

1. Baseline Data: This is data taken with essentially no shielding of the receiving antenna present. For tests requiring test fixtures, as in this test, the baseline data are collected with the EUT removed from the test fixture. For items such as enclosures the baseline data are collected with doors, access hatches, etc. removed.

2. Shielding Data: This is data taken with the paddle wheel and receiving antenna inside the EUT or test fixture with the EUT configured as nearly as possible for actual use. The difference between these data and the baseline data is the shielding effectiveness.

3. Dynamic Range Data: This is data taken to establish the maximum measurement range of the test setup. Expected leakage paths, through the EUT, are sealed up by wrapping suspected areas with aluminum foil, taping joints with aluminum tape, etc. The difference between these data and the baseline data should be greater than the difference between the shielding data and the baseline data. This ensures that the detected signal enters through the EUT and not through the test fixture or associated connectors, cabling, etc.

\section{Discrete Versus Noise Method}

\section{A. Discrete Method}

A block diagram of the measurement scheme used to perform the discrete frequency method of measuring shielding effectiveness is shown in Fig. 8. Before beginning the 


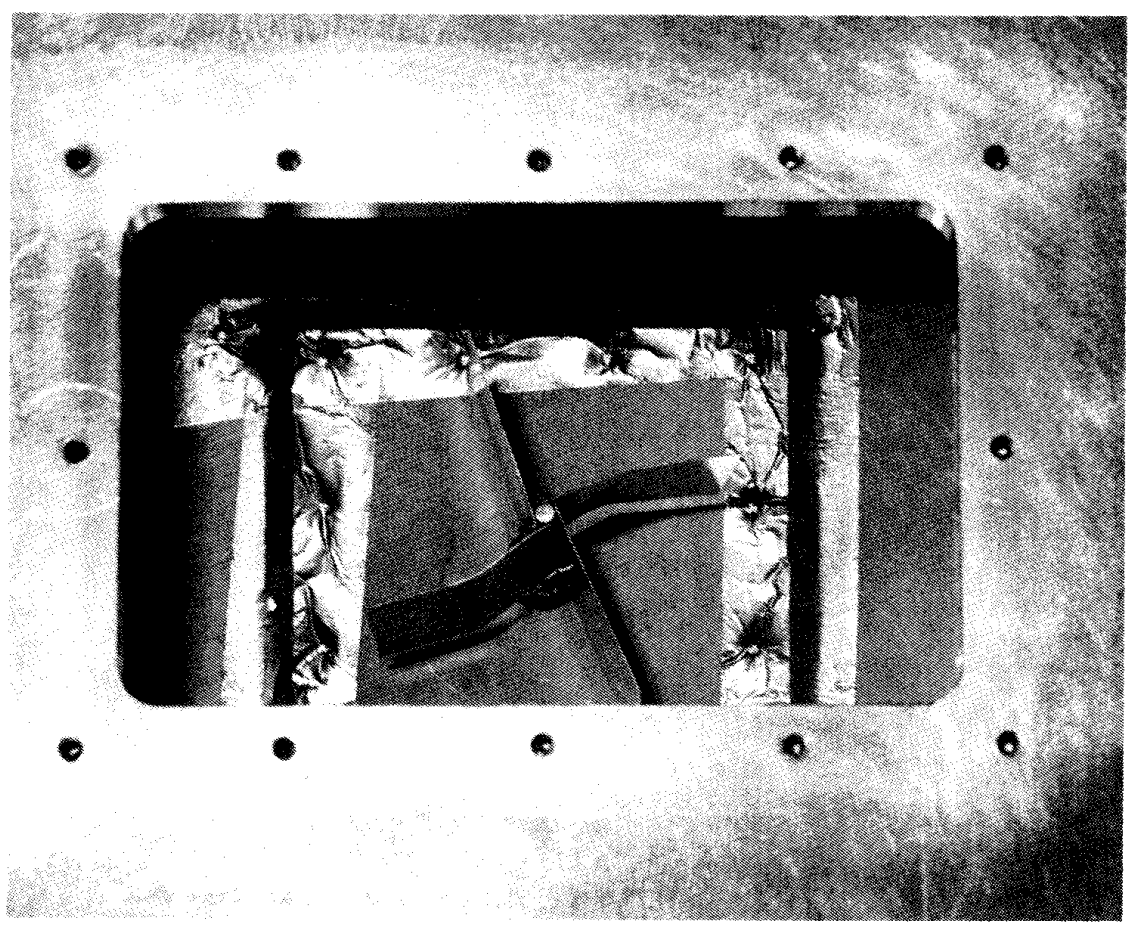

Fig. 6. Interior view of a test fixture.

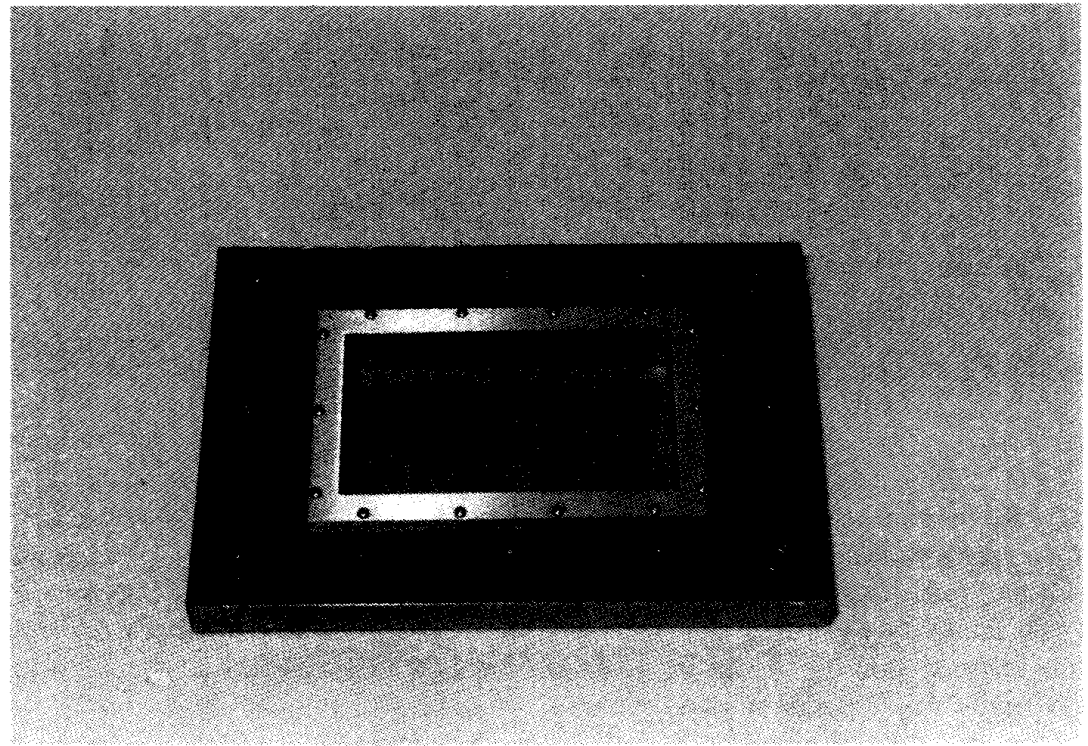

Fig. 7. Adaptor plate with EUT attached. 


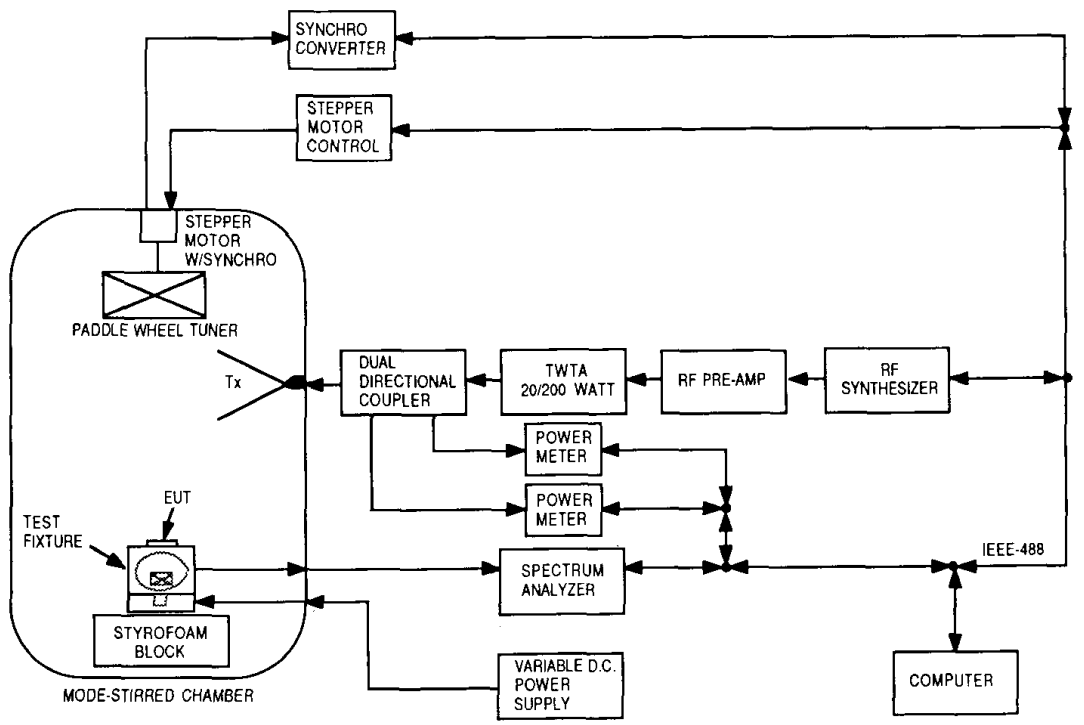

Fig. 8. Schematic arrangement used to perform the discrete frequency method of measuring the shielding effectiveness.

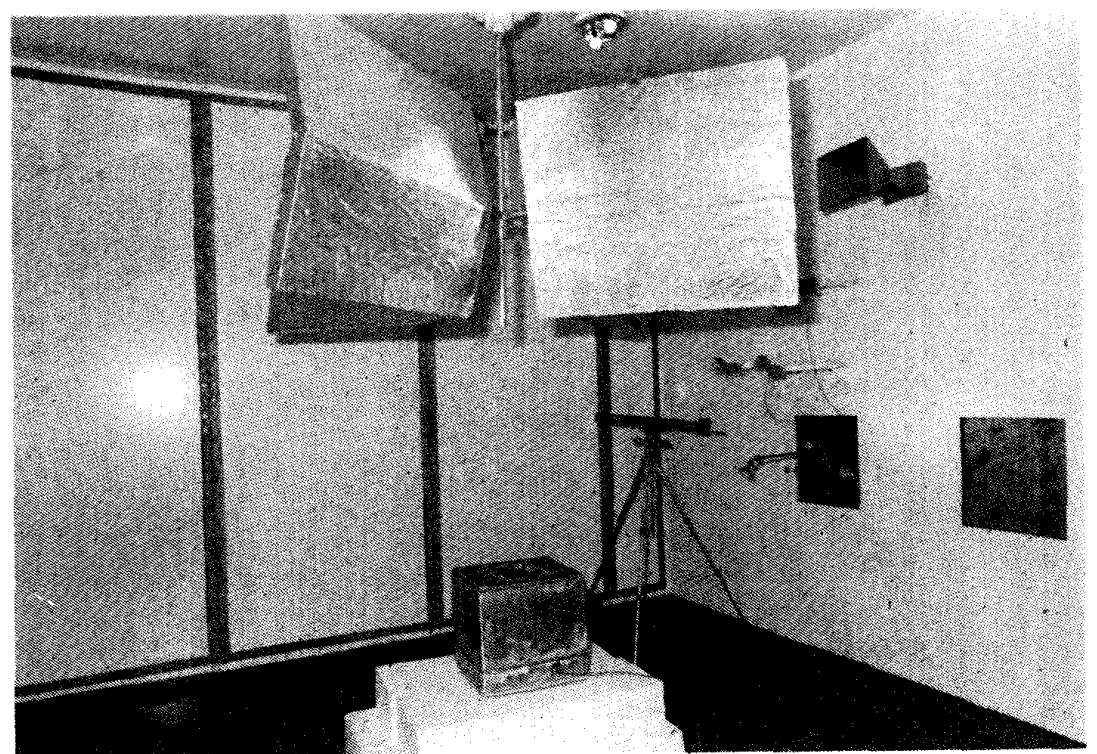

Fig. 9. Test fixture in chamber without the screen-window adaptor attached.

measurement sequence, the response time of the monitoring equipment, in this case a spectrum analyzer, and paddle-wheel rotation rates were determined. The response time of the spectrum analyzer was found by adjusting the spectrum analyzer to obtain the shortest possible sweep time while maintaining sufficient frequency resolution and dynamic range. This resulted in the following spectrum-analyzer settings: a) resolution bandwidth $=30 \mathrm{kHz}$; b) video bandwidth $=100 \mathrm{kHz} ;$ c) frequency span $=2 \mathrm{MHz}$; and d) sweep time $=0.02 \mathrm{~s}$. Starting with a very slow rotation rate, the internal paddle wheel was rotated progressively faster until a decrease in detected signal occurred. The fastest rate at which no decrease in detected signal occurred was found to be $40 \mathrm{r} /$ $\min (1.5 \mathrm{~s} / \mathrm{r})$. Therefore, $40 \mathrm{r} / \mathrm{min}$ was established as the rate of rotation of the internal paddle wheel. To insure one complete revolution of the internal paddle wheel per step of the external paddle wheel, a 2-s wait between steps was imposed. This yielded a measurement time of $6.7 \mathrm{~min}$ per frequency $(2 \mathrm{~s} \times 200 \mathrm{steps})$.

As previously stated, there are three measurements which must be made to insure valid shielding effectiveness measurements. The first is the collection of baseline data. Baseline data were obtained by placing the test fixture into the chamber without the screen window adaptor attached as shown in Fig. 9. Baseline data were collected under computer control using the measurement sequence outlined in Fig. 10. Next, the 


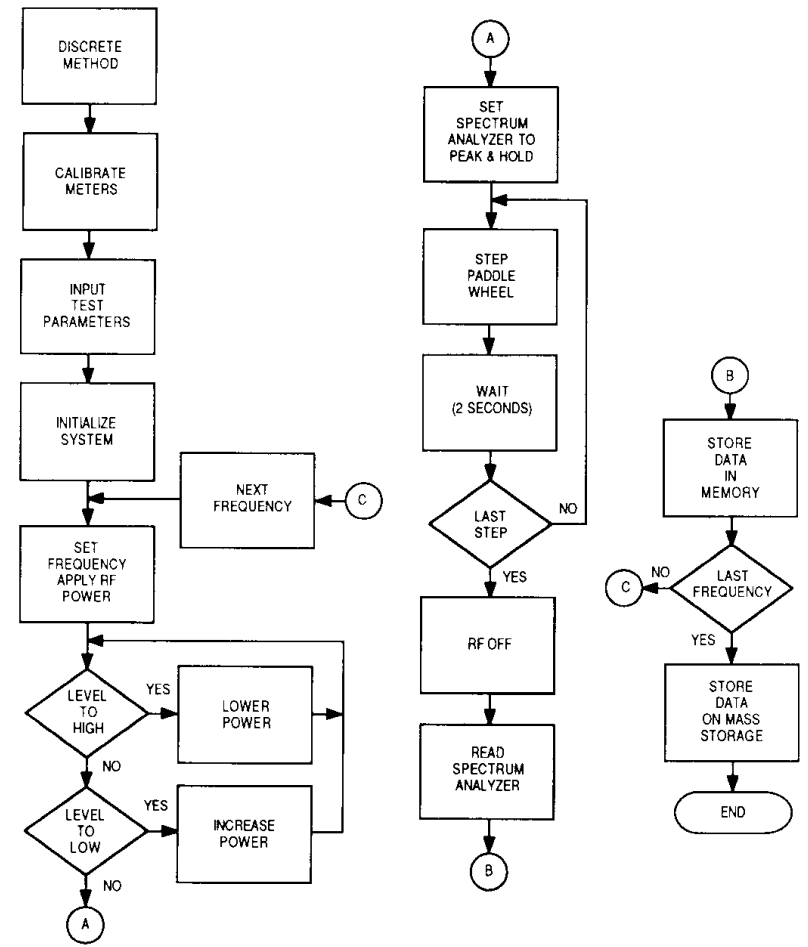

Fig. 10. Measurement sequence under computer control for collecting discrete data.

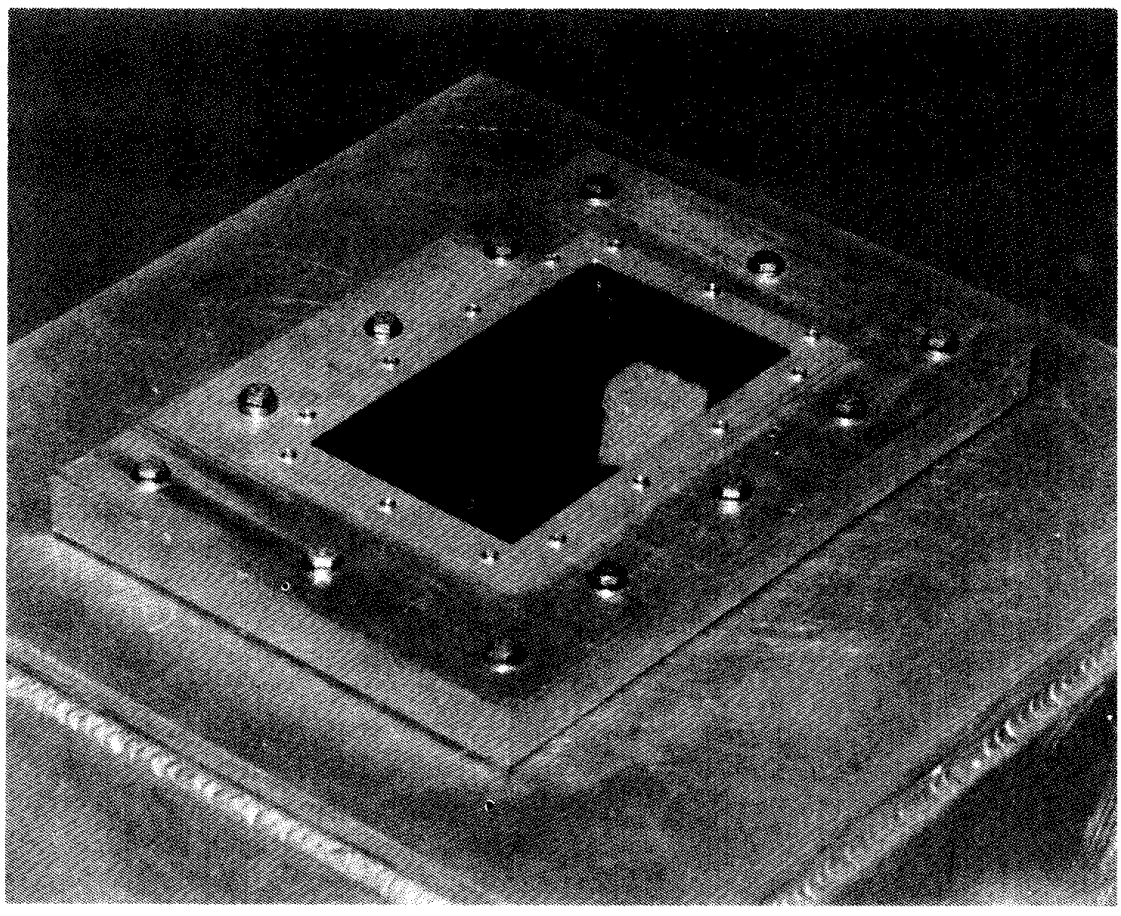

Fig. 11. Test fixture with a screen window.

adaptor with the screen window attached was mounted to the test fixture as shown in Fig. 11. The measurement sequence was repeated to collect the shielding data. The last step was to determine the dynamic range of the test setup. Aluminum foil was placed over the screen window and taped in place with aluminum tape as shown in Fig. 12. Care was taken to apply the foil and tape only to the screen window and adaptor plate and not to the interface between the adaptor plate and the test fixture. The measurement sequence was repeated to collect the dynamic range data. 


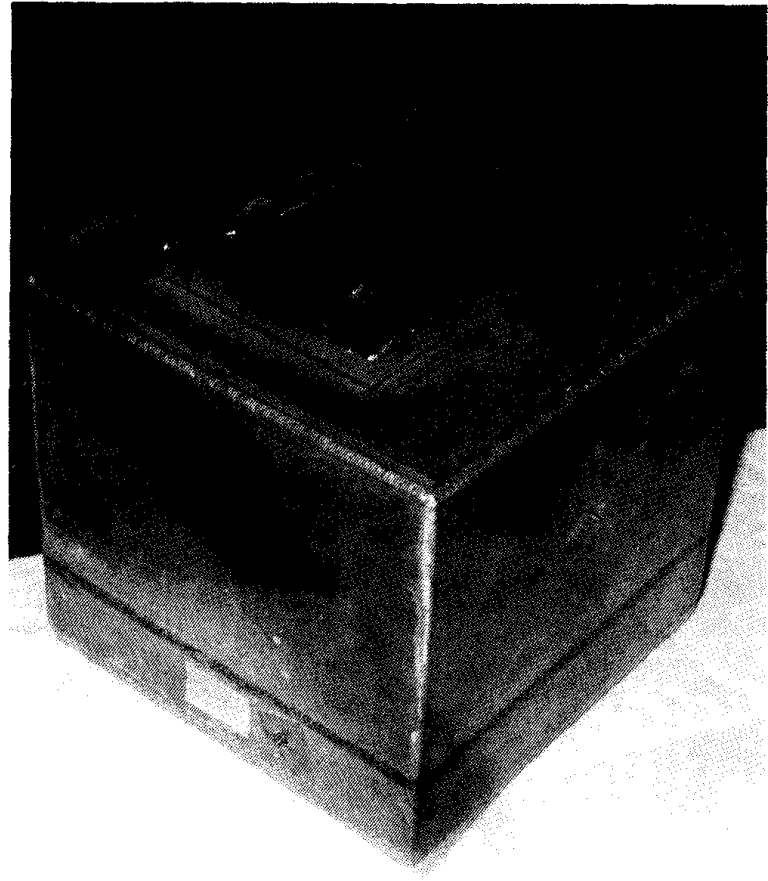

Fig. 12. Test fixture to determine the dynamic range.

TABLE 1

\begin{tabular}{ll}
\hline FREQUENCY BAND & FREQUENCY STEP \\
$1-2 \mathrm{GHz}$ & $250 \mathrm{MHz}$ \\
$2-4 \mathrm{GHz}$ & $250 \mathrm{MHz}$ \\
$4-8 \mathrm{GHz}$ & $500 \mathrm{MHz}$ \\
$8-18 \mathrm{GHz}$ & $500 \mathrm{MHz}$ \\
\hline
\end{tabular}

Testing was conducted in bands as listed in Table I. Results are shown in Fig. 13. The dynamic range shows an improvement in shielding of at least $20 \mathrm{~dB}$ for all frequencies tested, indicating that the measured shielding is that of the screen window and not test fixture leakage. Dynamic range data were not collected from 4 to $8 \mathrm{GHz}$ due to an equipment failure.

The amount of time spent collecting data is dependent upon the number of frequencies tested and the time required to rotate the external paddle wheel. For this test, each rotation of the external paddle wheel required $400 \mathrm{~s}$. Since each frequency required three separate data collection runs, the total test time per frequency was $1200 \mathrm{~s}$ or $20 \mathrm{~min}$.

\section{B. Noise Method}

A block diagram of the measurement scheme used to perform the noise method of measuring shielding effectiveness is shown in Fig. 14. Testing was limited to the 2-4-GHz band by equipment limitations. For the noise method, the amplified output of a solid-state avalanche noise diode was used to drive a 200-W TWT amplifier. Also, a preamplifier was added between the receiving antenna in the test fixture and the spectrum analyzer to amplify the received signal to a level

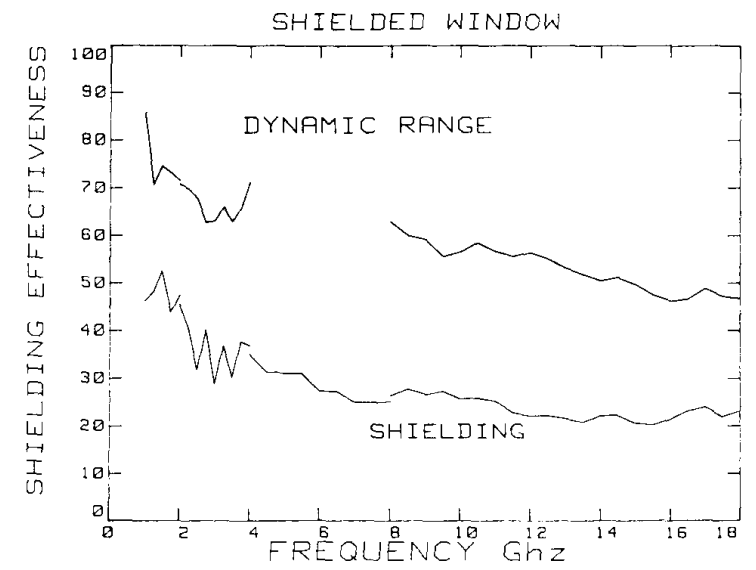

Fig. 13. Measured results of shielding effectiveness versus frequency.

which would permit a low sweep-time setting on the spectrum analyzer. To begin, as in the discrete method, the monitoring equipment response time and paddle-wheel rotation rates were determined. The response time of the spectrum analyzer was found by setting the spectrum analyzer to the desired start and stop frequencies and adjusting the spectrum analyzer to obtain the shortest possible sweep time while maintaining sufficient frequency resolution and dynamic range. This resulted in the following spectrum analyzer settings: a) resolution bandwidth $=1 \mathrm{MHz} ; \mathrm{b}$ ) video bandwidth $=100 \mathrm{kHz}$; c) sweep time $=$ $0.06 \mathrm{~s}$; d) start frequency $=2 \mathrm{GHz}$; and e) stop frequency $=$ $4 \mathrm{GHz}$. Starting with the previously established 2-s wait time between steps of the external paddle wheel, and leaving the rotation rate of the internal paddle wheel at $40 \mathrm{r} / \mathrm{min}$, the wait time for the external paddle wheel was progressively increased until no increase in received signal occurred. The shortest wait time at which no increase in detected signal occurred was found to be $12 \mathrm{~s}$. As before, baseline, shielding effectiveness, and dynamic range data were collected under computer control, this time using the measurement scheme outlined in Fig. 15. Test results are shown in Fig. 16.

Dynamic range data were again obtained by applying aluminum foil and aluminum tape to the screen window. The dynamic range results shown in Fig. 16 show an improvement in shielding for all frequencies tested. This indicates that the shielding measurement was not effected by test fixture leakage.

The spectrum analyzer used to collect the data for this paper was the digital type. The analyzer was capable of digitizing up to 1001 points or frequencies between the assigned start and stop frequencies. The frequency span for this test was 2000 $\mathrm{MHz}$ which yields a frequency resolution of approximately 2 $\mathrm{MHz}$. The external paddle wheel required $2400 \mathrm{~s}(12 \mathrm{~s} \times 200$ steps) to complete one revolution. Since three rotations were necessary to collect the required data, the total test time was $7200 \mathrm{~s}$ or $120 \mathrm{~min}$. Therefore, the total test time of $120 \mathrm{~min}$ resulted in a test time of only $7.2 \mathrm{~s}$ per frequency.

\section{Comparison: Noise Method Versus Discrete Method}

The results of the noise method show a great deal of fine structure not revealed by discrete frequency testing. Looking 


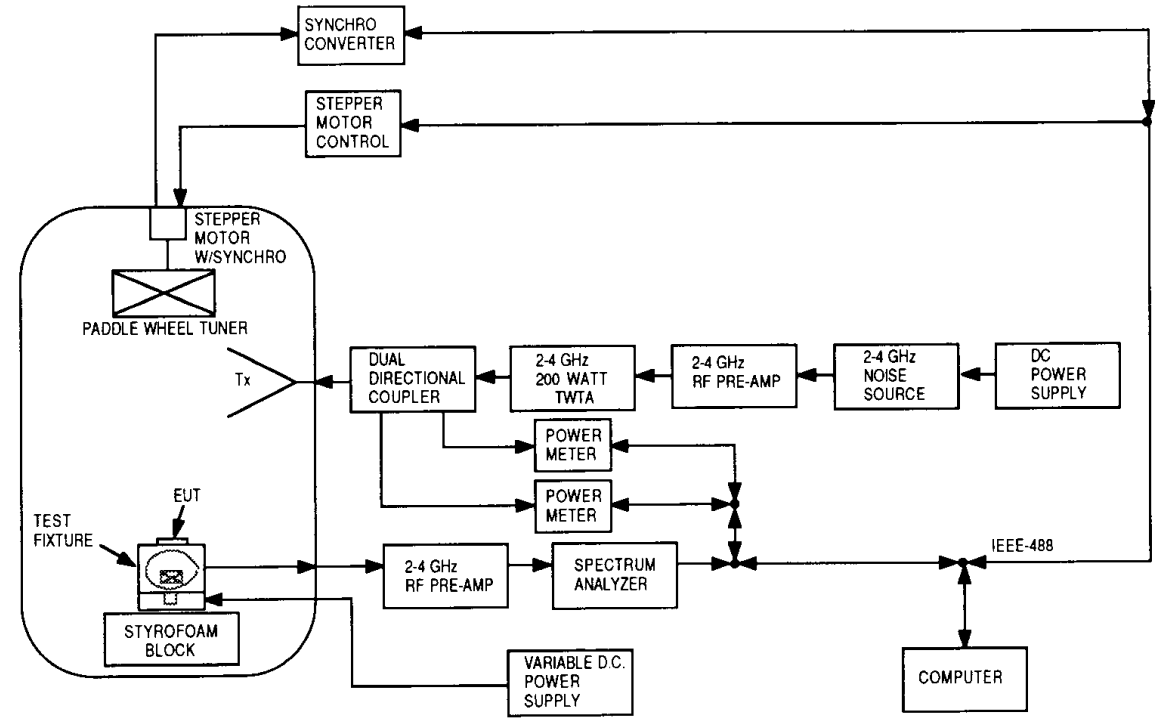

Fig. 14. Schematic arrangement use to perform the noise method of measuring the shielding effectiveness.

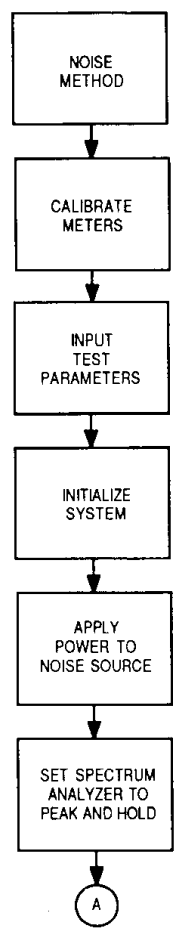

Fig. 15. Measurement sequence under computer control for collecting noise data.

at Fig. 17 we see the results of the noise method plotted against discrete frequency data at $250-\mathrm{MHz}$ intervals. An examination of the area highlighted by the box in Fig. 17 is shown in Fig. 18 in which the discrete frequency data are spaced at $25-\mathrm{MHz}$ intervals. The agreement between the noise data and discrete data is quite good; however, there is still a lot of detail between the points which needs to be examined. Fig. 19 shows an expanded view of the area outlined by the box in Fig. 18.

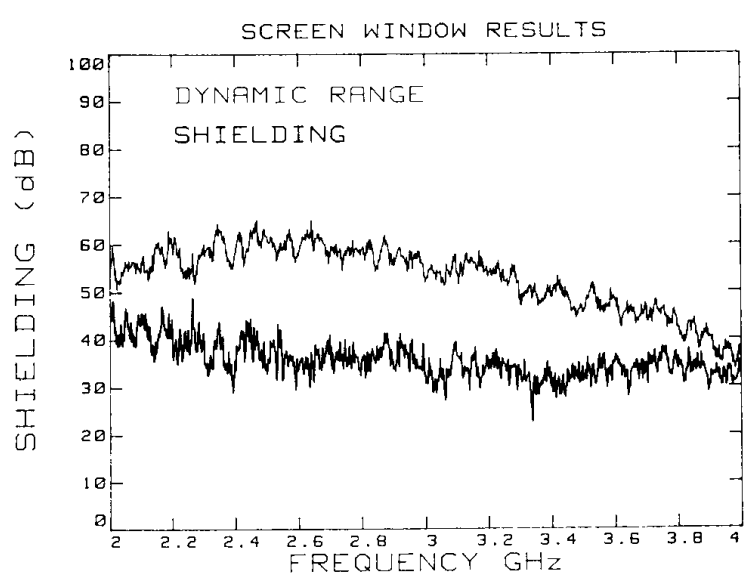

Fig. 16. Measured results versus frequency using the noise method.

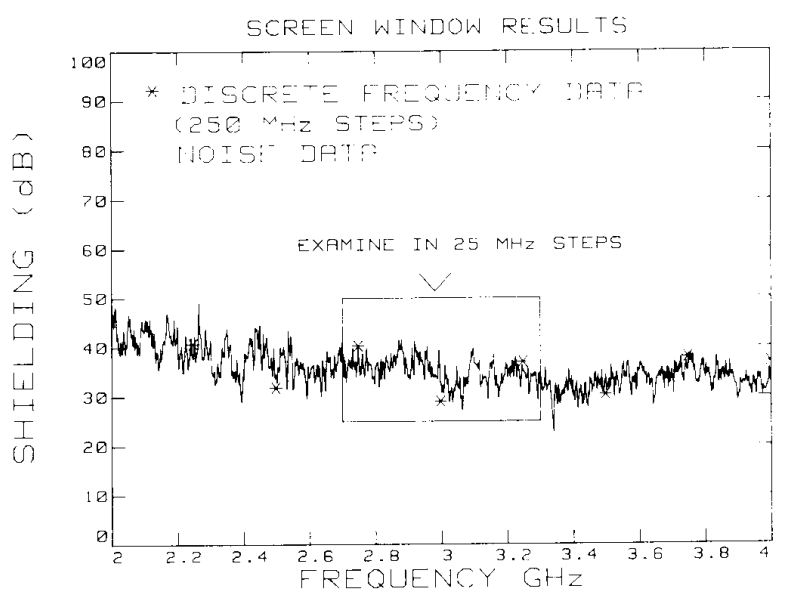

Fig. 17. A comparison of results obtained by both methods. 


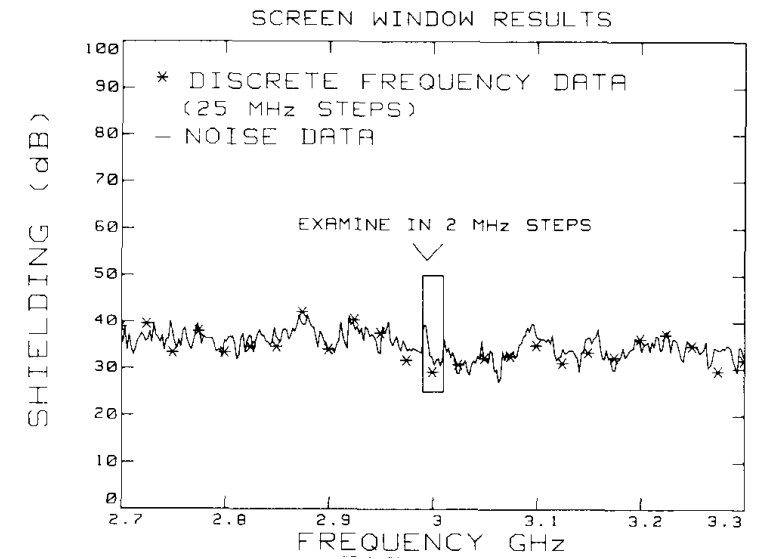

Fig. 18. Detailed comparison of results obtained by both methods, with the discrete frequency data taken at $25-\mathrm{MHz}$ intervals.

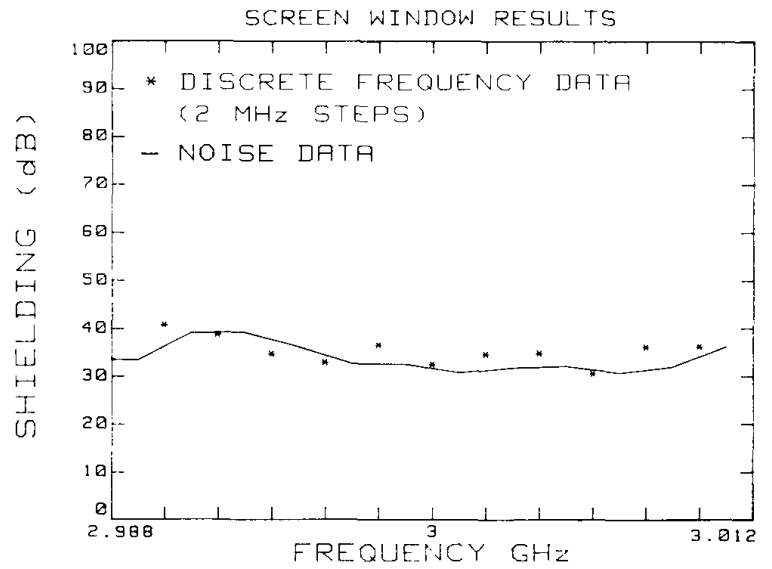

Fig. 19. Detailed comparison of results obtained by both methods, with the discrete frequency data taken at $2-\mathrm{MHz}$ intervals.

The discrete frequency data in Fig. 19 are spaced at $2-\mathrm{MHz}$ intervals. It can be seen that the noise data highlighted in Fig. 18 are closely approximated by the discrete frequency data of Fig. 19.
The time required to perform a discrete frequency test, for the case presented in this paper, was 20 min of test time per frequency. The time required to perform a noise test, for the case presented in this paper, was $7.2 \mathrm{~s}$ per frequency.

The dynamic range of the discrete frequency method is greater than the noise method due to the ability to concentrate energy at discrete frequencies. Using low-power/high-gain amplifiers to amplify the received signals of the noise method will increase the dynamic range of the noise method, but not to the point of being equal to the discrete method for a given amount of output power.

\section{Conclusions}

For a given test time the noise method offers a significant increase in the number of frequencies tested over the discrete frequency method, but at a loss in dynamic range. The use of relatively inexpensive noise sources and low-power broadband amplifiers offers a fast, economical, and repeatable method of performing radiated shielding effectiveness measurements over large frequency spans in short periods of time.

\section{ACKNOWLEDGMENT}

The author wishes to thank J. L. Bean of NSWC and L. C. Halley of EG\&G, Washington Analytical Services Center Inc., Dahlgren, VA, for their support in preparing this paper.

\section{REFERENCES}

[1] J. D. Lee, "MIL-STD-1377 vs. MIL-STD-285 microwave shielding effectiveness measurements," in 1975 IEEE EMC Symp. Rec., p. 4BIf $1-4$.

[2] M. L. Crawford and G. H. Koepke, "Design, evaluation, and use of a reverberation chamber for performing electromagnetic susceptibility/ vulnerability measurements," NBS Tech. Note 1092, Apr. 1986.

[3] W. Jarva, "Shielding tests for cables and small enclosures in the 1-10 GHz range," IEEE Trans. Electromagn. Compat., vol. EMC-12, no. 1 , p. 12 , Feb. 1970.

[4] J. L. Bean and R. A. Hall, "Electromagnetic susceptibility measurements using a mode-stirred chamber," NBS NBSIR 81-1638, Feb. 1981.

[5] M. L. Crawford and G. H. Koepke, "Comparing EM susceptibility measurements between reverberation and anechoic chambers," presented at the IEEE Int. Symp. EMC, Boston, MA., Aug. 20-22, 1985

[6] R. E. Richardson, "Mode-stirred chamber calibration factor, relaxation time, and scaling laws," IEEE Trans. Instrum. Meas., vol. IM34, no. 4, pp. 573-580, Dec. 1985. 